Acta Hispanica (2020) Supplementum II: 367-375

\title{
POBREZA Y PROGRAMAS SOCIALES EN MÉXICO
}

\author{
VERónica VILLARESPE REYES \\ Instituto de Investigaciones Económicas, UNAM \\ Bernardo Ramírez Pablo \\ Instituto de Investigaciones Económicas, UNAM
}

Carlos Quintanilla Yerena

Facultad de Derecho, UNAM

\begin{abstract}
Resumen: Del siglo XVI al XIX, aproximadamente, la concepción de la pobreza tenía un fuerte contenido moral. La pobreza de quienes estaban sanos y no trabajaban provenía de la lujuria, el vicio, la pereza y la arrogancia. Es por ello que el trabajo adquiere un carácter rehabilitador. Así, el problema de la pobreza ha estado presente, en el mundo occidental, a través de casi cinco siglos, tanto como la preocupación de cómo aliviarla, de qué hacer con los pobres. El contenido de la pobreza, así como su concepción han cambiado a través de las épocas históricas. La aparición de programas contra la pobreza es contemporánea y pasa por los lineamientos que proponen los organismos internacionales, principalmente el Banco Mundial. En México, por ejemplo, los programas de transferencias monetarias condicionadas se instrumentaron a partir de 1997, nos referimos a Progresa-Oportunidades-Prospera. La pobreza es funcional al sistema, por ende, entonces no ha podido ni puede ser cancelada por un programa. Así, muchos esfuerzos se han hecho para aliviar la pobreza, nunca para cancelarla.
\end{abstract}

Palabras clave: pobreza, concepción de la pobreza, programas de transferencias monetarias condicionadas.

\begin{abstract}
From the 16th to the 19th century, approximately, the conception of poverty had a strong moral content. The poverty of those who were healthy and did not work came from lust, vice, laziness and arrogance. That is why the work acquires a rehabilitative character. Thus, the problem of poverty has been present, in the western world, for almost five centuries, as well as the concern of how to alleviate it, of what to do with the poor. The content of poverty, as well as its conception have changed throughout historical times. The emergence of anti-poverty programs is contemporary and goes through the guidelines proposed by international organizations, mainly the World Bank. In Mexico, for example, the conditional cash transfer programs were implemented as of 1997, we refer to Progresa-Oportunidades-Prospera. Poverty is functional to the system, therefore, it could not and cannot be canceled by a program. Thus, many efforts have been made to alleviate poverty, never to cancel it.

Keywords: Poverty, Conception of Poverty, Conditional Cash Transfers Programs.
\end{abstract}


Pobreza y programas sociales en México

\section{El fenómeno de la pobreza. Preocupación mundial}

En la actualidad, la preocupación por la existencia y persistencia de la pobreza ocupa un lugar relevante en las agendas nacionales de desarrollo y en las reuniones de organismos internacionales, como el Banco Mundial. La pobreza se interpreta dentro de un marco teórico-histórico, ¿quiénes son los pobres y por qué son pobres? En otras palabras, el contenido de la pobreza, su conceptualización y su alivio cambian de acuerdo con la época histórica y al pensamiento teórico y político dominante. Sin embargo, la cuestión fundamental por la que habríamos de empezar es en qué momento histórico la pobreza se convierte en problema. En el feudalismo, por ejemplo, la pobreza era vista como algo natural, no como un problema social ni económico; la pobreza rara vez se concebía como un valor absoluto: era algo relativo, una cualidad de impecunio, de enfermedad, de infortunio, pena, desprecio, disgusto.

Desde el siglo XVI hasta fines del siglo XVIII la concepción de la pobreza giraba en torno a la dignidad e indignidad de los pobres: quienes querían trabajar, pero no podían (porque no eran capaces de hacerlo) eran dignos de recibir ayuda; quienes no querían trabajar, pero sí podían hacerlo, eran indignos de ser ayudados. La ayuda se otorgaba mediante Leyes especiales, conocidas como las Leyes de Pobres ${ }^{1}$. Esta concepción prevaleció en particular en Inglaterra, país en el que se inicia, con más claridad, el tránsito del feudalismo al inicio del capitalismo mercantil y fue la dominante en el mundo occidental cristiano.

Para el naciente régimen mercantilista era necesario tener y fomentar una población numerosa y trabajadora que permitiera mantener bajos salarios (Stephenson, 1965), para abastecer a las nacientes manufacturas que a su vez producirían cuantiosas mercancías para el comercio exterior, trayendo metales preciosos a las arcas de la nación respectiva y con ello la prosperidad. La utilidad de la pobreza se centraba, pues, en que un salario real debería significar un "nivel óptimo de frustración", es decir, debía ser suficientemente alto para proporcionar un incentivo para los "lujos" y suficientemente bajo para que nunca pudieran alcanzarse (Stephenson, 1965).

El trabajo poseía un fuerte componente moral: se consideraba rehabilitador, una obligación esencial que debían cumplir quienes estaban capacitados para él. A finales del siglo XVII, la concepción del trabajo se va transformando, de medio para conseguir la redención a través de la autodisciplina hacia instrumento apropiado para la corrección punitiva. Redención y castigo se complementaban, castigo para el que no trabaja y pan para quien no puede hacerlo.

\footnotetext{
${ }^{1}$ En Inglaterra se dictan las primeras Leyes de Pobres en el siglo XVI: The Statute of Legal Settlement, (1547), An Act for Setting of the Poor on Work and for the Avoiding of Idleness, (1576), An Acte for the Reliefe of the Poore (1597-1598). Finalmente, en esta última Ley pero del año 1601 (Estatuto 43, capítulo 2) se define y establece claramente la dignidad e indignidad de los pobres. Véase Villarespe Reyes, Verónica (2002). Pobreza: teoría e historia. México: Juan Pablos-IIEc-UNAM. 16-17 y 21 nota al pie número 14 .
} 
Con la Revolución Industrial, a mediados del siglo XVIII, la población total sufrió un incremento considerable debido, principalmente, a que descendió la mortandad (sobre todo por el descubrimiento de la vacuna contra la viruela); la mejora en las técnicas agrícolas, que aumentaron la disponibilidad de alimentos y la introducción del algodón en la confección y tuberías baratas para suministrar el agua y para el drenaje, incidiendo en un mayor grado de higiene. Sin embargo, estas mejoras fueron acompañadas por masivas oleadas de indigencia y un deterioro en las condiciones de vida de la clase obrera: recuérdense las condiciones en las que se desenvolvía el trabajo infantil y el trabajo de las mujeres (Derry-Williams, 1980: 401; Marx-Engels, 1971). Se empezó a debatir si la ayuda a los pobres, más allá de aliviar su necesidad, los inducía a la pereza y al vicio. Se crearon pobres con diferentes características, y se empezó a identificar la pobreza como problema social.

El desarrollo de la Revolución Industrial introdujo la discusión sobre el progreso, y con ello la distinción entre pobreza relativa y pobreza absoluta. Destacan en este sentido, las posiciones antagónicas de Adam Smith y de Thomas Robert Malthus. Para Smith, el poder del trabajo era la causa de la riqueza; para Malthus, el poder de la pobreza era la causa del trabajo; para Smith, la característica básica de los pobres es que eran trabajadores; para Malthus la característica del trabajador era su pobreza, pues sin ella carecería de motivos para trabajar. Para Smith, la pobreza era relativa porque por el progreso económico los muy pobres se convertirían en pobres, y los menos pobres vivirían en una confortable pobreza. Para Malthus, la pobreza tendía a ser absoluta, pues el pobre sería muy pobre y los muy pobres llegarían al hambre y hasta la muerte. Smith era "optimista", Malthus "pesimista” (Villarespe Reyes, 2000: 29-30).

\section{2. ¿Ayuda a los pobres o cancelación de la pobreza?}

Después de 1945 las Leyes de Pobres, como política social, fueron sustituidas por el estado del bienestar ${ }^{2}$. Es precisamente a partir de la Segunda Guerra Mundial, que se perfila otra forma de conceptualizar la pobreza, ya que ésta no había disminuido con el desarrollo del mercado -como la teoría y la ideología dominante auguraron-, y tampoco fue elemento transformador. En particular, cobran relevancia los conceptos de desarrollo y de subdesarrollo, que realmente parten al mundo en dos mundos: el de países ricos y el de países pobres. Aunque la pobreza existe tanto en unos como en otros, en los países

\footnotetext{
${ }^{2}$ Sobre la discusión de los orígenes del Estado del Bienestar, véase Salazar Silva, Fernando (2005). La configuración del Estado de Bienestar. Elementos constitutivos. Reflexión Politica, 14. "En los distintos momentos históricos el Estado de Bienestar ha cumplido acciones diferentes. Cabe resaltar el papel que juega antes de la revolución industrial con un carácter eminentemente caritativo y logra establecer el orden social para convertirse más tarde en el baluarte de la democracia y del crecimiento económico. Es claro que las ideas y los mecanismos de beneficencia y seguridad social se fueron refinando en Europa incluso desde las instancias medievales" (138). Véase también Himmelfarb, 1998: 13-14.
} 
subdesarrollados la pobreza es más acentuada y lacerante y adquiere dimensiones realmente dramáticas ${ }^{3}$.

El problema de la pobreza ha estado presente a través de casi cinco siglos, así como la preocupación de cómo aliviarla, de qué hacer con los pobres. Como Charles Booth (1889) y Seebohm Rowntree (1901) demostraron, la pobreza involucra supuestos objetivos, susceptibles de ser medidos, y otros subjetivos (por ejemplo, los vicios). Es lugar común que se midan los ingresos de unos individuos respecto de otros, y de allí se derive el nivel de vida que tienen y al que se aspira que logren, mediante políticas o programas adecuados a tal fin. En la concepción dominante contemporánea de la pobreza, ser pobre es carecer de los recursos y servicios para satisfacer las necesidades básicas y participar de lleno en la sociedad (Drèze-Sen-Hussein, 1995: v-vi).

La propuesta de Amartya Kumar Sen, de reemplazar ingreso y utilidad por la idea de capacidad, ha dado un vuelco teórico y político a la teoría de la elección colectiva y bienestar social: capacidad representa las combinaciones alternativas que una persona puede hacer o ser y/o distintos funcionamientos que puede alcanzar (Nussbaum-Sen, 1998: 67-69). A la satisfacción en la extrema urgencia de una clase de necesidades básicas, se le ha asignado una importancia moral y política particular. Sen ha utilizado el término capacidades básicas con el objeto de separar la habilidad para satisfacer ciertos funcionamientos crucialmente importantes hasta ciertos niveles adecuadamente mínimos. La identificación de niveles mínimos aceptables de ciertas capacidades básicas, por debajo de los que se consideran padecimientos de privaciones escandalosas, proporciona un enfoque de la pobreza: la identificación de la combinación mínima de capacidades básicas es una buena forma de plantear el problema del diagnóstico y la medición de la pobreza.

En la visión de Sen, la calidad de vida debe evaluarse en relación con la capacidad para lograr funcionamientos valiosos. $\mathrm{Y}$ a su vez, las capacidades humanas constituyen una parte importante de la libertad individual. La lucha contemporánea contra la pobreza pasa por establecer y elegir grupos de personas específicos que se caracterizan por bajo consumo, desnutrición, bajos niveles educativos, malas condiciones sanitarias, participación inestable en los sistemas productivos, actitudes de desaliento o anémicas, baja participación en los mecanismos de integración social, y adhesión a una escala de valores diferente a la del resto de la sociedad. Estos grupos serán los sujetos de los programas contra la pobreza. Programas de enfrentamiento a la pobreza apoyados por el Banco Mundial como el Programa de Educación, Salud y Alimentación (ProgresaOportunidades-Prospera) en México, derivan su filosofía y acción normativa en aumentar las capacidades de los pobres: alimentación, salud y educación, les proporcionarán funcionamientos adecuados para una calidad de vida mejor.

\footnotetext{
${ }^{3}$ Llama la atención que en este siglo XXI con todo el desarrollo de las fuerzas productivas que se ha concretado en adelantos torales, la población más afectada por la indigencia se compone de mujeres solas -viudas y madres solteras-, niños y ancianos, al igual que siglos antes.
} 
Muchos esfuerzos se han realizado -y se realizan- para enfrentar la pobreza, en nuestros días con programas específicos, sin embargo, sus propósitos no han sido cancelarla, sino paliarla, aliviarla, mitigarla. Además, cumplen con un objetivo políticosocial primordial: siguiendo a Sen la mejoría de la condición humana es más que una proposición humanitaria justa, es una necesidad de sobrevivencia, para todos los desposeídos y los no desposeídos. El peligro estriba que un orden político no estable puede ser construido sobre las bases de sociedades perturbadas a causa de la pobreza extrema (Drèze-Sen-Hussein, 1995).

Las diferencias sociales y económicas entre países y entre clases e individuos se acentúan cada vez más, con todo y a pesar de los gigantescos logros científicos, de la tecnología necesaria, del conocimiento de las fuentes y transformación de las materias primas, y las habilidades y destrezas para satisfacer las necesidades primordiales. Podría suponerse entonces que los alimentos, por ejemplo, deberían alcanzar para todos, pero no todos tienen acceso a ellos. Como Amartya Kumar Sen reconoce, nunca como antes hemos fallado en hacerlo y la persistente masa de pobres es parte de la vida del siglo XXI, como agregamos nosotros lo ha sido desde hace varios siglos.

\section{Focalización y programas contra la pobreza}

La CEPAL propuso, en la década de 1990, la revisión del sistema general de subsidios y los instrumentos de la política social, con el ulterior propósito de racionalizar el gasto social (CEPAL, 1995: 13-28). Focalizar como condición necesaria para racionalizar, evaluar la relación costo-impacto de cada programa, incorporar a otros actores sociales (organismos no gubernamentales, municipios, comunidades, beneficiarios, y empresas privadas) como ejecutores y si es posible como financiadores de los programas, y priorizar la demanda de servicios para catalizar la relación de la política social del Estado con la sociedad civil $^{4}$, constituyeron los ejes alrededor de los que girarían los programas de transferencias monetarias condicionadas contra la pobreza.

La focalización será ya indispensable en tanto que permitirá concentrar los recursos disponibles en una población de beneficiarios potenciales, que se identificará para atender un problema determinado o una necesidad definida. Es decir, se segmentará a la población para elevar el impacto o beneficio potencial per cápita, en tanto que en el enfoque homogéneo no se consideran diferencias y peculiaridades de la población a beneficiar.

La focalización es un criterio de inclusión, pero también de exclusión ${ }^{5}$, en tanto que se basa en la segmentación del mercado, y sus objetivos políticos fundamentales son

\footnotetext{
${ }^{4}$ Esto se enfatiza en el documento presentado por la Secretaría de la CEPAL en la III Conferencia Regional sobre la Pobreza en América Latina y el Caribe (Santiago de Chile, noviembre 1992).

${ }^{5}$ Consideramos que la "modernidad" del criterio de focalización no es tal, en tanto que en política social siempre han existido determinaciones sobre las ahora llamadas poblaciones-objetivo: los vagos y los mendigos que no estaban sanos y no podían trabajar, aunque quisieran hacerlo,
}

Acta Hispanica, Hungría, Supplementum II: 367-375, 2020, ISSN: 1416-7263 | 371 
devolver la credibilidad al Estado e instrumentar mecanismos para disminuir el clientelismo. Se afirma que la selección de prioridades en política social está relacionada con el trato preferencial de problemas identificados por un gobierno y con la postergación de otros, pero, además, agregamos nosotros los problemas no sólo se identifican, sino que se califican políticamente. Ello implica que el gobierno opta por realizar obras físicas, productivas o de carácter social, eligiendo a cuál o a cuáles rubros orientará la inversión (educación, salud o vivienda) y las diversas formas en que lo hará. Fija qué monto otorgará a cada política y determina a los beneficiarios, lo que involucra que ciertos grupos de la población pueden ser focalizados o designados como objetivos de la política social, quedando al margen otros (Hernández-Orozco-Vázquez, 2005; Villatoro, 2004: 13-27) ${ }^{6}$. Racionalización y eficiencia son los ejes de la concepción cepalina en política social, apoyada en los lineamientos del Banco Mundial.

\section{Programas contemporáneos en México: Progresa, Oportunidades y Prospera}

En 1997 surge el Programa de Educación, Salud y Alimentación (Progresa) y fue renombrado en 2002 Programa de Desarrollo Humano Oportunidades. Posteriormente en 2014, se convierte en Prospera, programa de inclusión financiera, vigente hasta 2019, dejando de lado programas como Solidaridad que privilegiaban el desarrollo económico para enfrentar la pobreza. Esto se explica porque los organismos, dominantes en la "lucha contra la pobreza", cambiaron de dictados, de postulados y de rumbos. A fines de la década de 1990, el Banco Mundial da un giro en sus propuestas en la lucha contra la pobreza y se instrumentan los programas de transferencias monetarias condicionadas, de los cuales el Progresa-Oportunidades-Prospera es representativo en México. Estos utilizan la focalización geográfica, y otorgan recursos monetarios a las familias-objetivo y ciertos servicios suponiendo que como la pobreza se hereda, se rompe así el círculo vicioso de la pobreza y ésta ya no se reproducirá generacionalmente. Sus objetivos centrales se resumen en: 1) mejorar las condiciones de alimentación, salud y educación,

obligados a ingresar en los asilos-talleres; los ancianos, albergados en los hospicios y asilos; los niños huérfanos y los expósitos, recogidos en casas de misericordia; las viudas ingresadas en conventos, etc.

6 "Estadísticamente es inevitable la presencia de errores en la focalización y los responsables de implementar la política social tienen que decidir entre incurrir en un error de inclusión o de exclusión cuando se elige un método. Un error de inclusión desperdicia recursos del programa o hace menos eficientes sus resultados, al incluir a beneficiarios con ingresos mayores a la línea de pobreza, mientras que un error de exclusión limita el cumplimiento del objetivo de reducir la pobreza pues no incorpora a los individuos que realmente lo necesitan. Cuando se compara entre métodos, aquellos con un buen desempeño en tasas de subcobertura generalmente no son tan buenos en términos de reducir la tasa de fuga" (Hernández-Orozco-Vázquez, 2005: 56). Tasa de fuga: población no meta beneficiaria del programa; tasa de subcobertura: población meta que no es alcanzada por el programa. 
en particular de los niños y de sus madres, que impacten un mejor aprovechamiento escolar y abatan la deserción escolar; 2) apoyar a la economía familiar "procurando que el hogar disponga de recursos suficientes para que los hijos completen su educación básica"; 3) hacer corresponsables y partícipes a todos los miembros de la familia en acciones de bienestar social; y 4) promover la participación y el respaldo comunitario a las acciones que se emprendan, para que los servicios educativos y de salud beneficien al conjunto de las familias.

\section{Reflexiones}

Los programas de transferencias condicionadas parten de un enfoque integral, en el que a mayor nivel de educación el uso de los servicios de salud mejora, favoreciendo la higiene y la prevención; la salud infantil y juvenil incide en un mayor y mejor rendimiento escolar; y un mejor estado nutricional coadyuva a desarrollar las capacidades de las personas. Estos factores se imbrican entre sí y a un largo plazo la hipótesis oficial es el trocamiento del círculo vicioso de la pobreza en círculos virtuosos de combate a la pobreza.

En una óptica meramente subjetiva e individualista, se afirma que "la superación de los factores que dan lugar a y perpetúan la condición de pobreza depende fundamentalmente de la activa corresponsabilidad de las familias beneficiarias y sus comunidades" (Progresa, 15-06-2019). Además, se ha argumentado que estos programas no desalientan la autoayuda, y en ese sentido se establecen los montos de las transferencias mensuales (Scott, 1999; Programa de desarrollo, 2007). Aquí encontramos en el fondo la vieja discusión que se dio en Inglaterra, en los siglos XVII y XVIII, acerca de si la ayuda a los pobres desalentaba su interés por ser empleados, por trabajar, como si el ser empleado, el trabajar, dependiera de ellos y no de condiciones externas y ajenas a ellos. En este sentido, como hemos expuesto al principio de este texto, el debate se centraba en los pobres meritorios de la ayuda y en los que no la merecían: castigo para el que no trabajara y pan para el que no pudiera hacerlo.

Los programas de tipo conditional cash transfers se insertan en la concepción de Milton Friedman, representante de la Segunda Escuela de Chicago que se inició en la década de 1960. Para Friedman, si el Estado ayuda a los pobres deberá hacerlo en metálico, pues así se establece claramente lo que esa ayuda le cuesta a la sociedad. De acuerdo con su defensa de la "libertad", plantea que el recurso más conveniente para la "eliminación" de la pobreza, es la caridad, en tanto que ésta es voluntaria y no obligatoria (Sugden, 1982: 341). Aunque pudiera aceptar, dice, la acción del Estado en dicha eliminación para establecer un mínimo en el nivel de vida de cada miembro de la sociedad, estaría a discusión "cuánto y cómo hacerlo": a) si el objetivo es el alivio a la pobreza se debe tener un programa encaminado a ayudar al pobre ("ayudar a la gente como gente") y b) dicho programa "no debería deformar el mercado o impedir su funcionamiento, si es que ha de establecerse a través del mercado" (Friedman, 1966: 242-243). También propone graduar 
la escala de subsidios y de impuestos, sobre la exención, para fijar un mínimo de ingreso debajo del cual no se encuentre ningún miembro de la sociedad, después de incluir el subsidio. Ese mínimo estaría en relación con la capacidad de financiamiento de la sociedad, en otras palabras, así se establecerá claramente lo que le cuesta a la sociedad la ayuda a los pobres.

A riesgo de obviar, consideramos que Progresa-Oportunidades-Prospera son caridad institucionalizada, pues las ayudas monetarias que otorgan no transforman las condiciones en las que se origina y desarrolla la pobreza, pero sí manifiestan a la sociedad cuánto cuesta luchar contra ella. Reiteramos lo que hemos sostenido en diversas ocasiones y en otros textos: la pobreza se hereda y su círculo vicioso no puede convertirse en uno virtuoso, pues la pobreza se reproduce, dentro del mismo sistema que la engendra. El mismo Gary Becker (1974), teórico del capital humano señala que: “[...] Por ejemplo, el capital humano de una persona es la suma de la cantidad heredada y la que adquiere a través de inversiones; más aún, la cantidad invertida es particularmente determinada por la herencia" (1070). Los pobres generalmente provienen de familias pobres que en su mayoría desdeñan la educación, en aras de la premura porque sus hijos generen ingresos inmediatos para la subsistencia del hogar; ello se convierte en un freno para la movilidad social intergeneracional, eliminando la posibilidad de que los hijos se vinculen al mercado laboral con una mejor remuneración. Por todo lo anterior, se hace necesario que la política económica promueva una efectiva y eficiente inclusión en el mercado laboral (Aguado-Girón-Gary, 2007: 452-460).

Se trata más bien de programas asistencialistas, ya que aparecen ajenos a otras esferas de la política económica; tratan a los pobres como si estuvieran fuera de la producción, la distribución, el intercambio y el consumo, es decir como si los pobres estuvieran aislados del sistema que los procrea y no inmersos y viviendo dentro de él como están.

\section{Referencias bibliográficas}

Aguado, Luis Fernando-Girón, Luis Eduardo-Salazar, Fernando (2007). Pobreza y educación urbanas en el valle del Cauca, Colombia. Revista Comercio Exterior, 6. 448-461. Becker, Gary S. (1974). A theory of social interactions. The Journal Political Economy, 6. 1063-1093.

CEPAL (1995). Focalización y pobreza, Santiago de Chile: Naciones Unidas. Cuadernos de la CEPAL, 71.

CEPAL (1992). III Conferencia Regional sobre la Pobreza en América Latina y el Caribe. Santiago de Chile.

Derry, T. K.-Williams, Trevor I. (1980). Historia de la tecnología, desde 1750 hasta 1900 (I/2). Madrid: Siglo Veintiuno. 
Verónica Villarespe Reyes - Bernardo Ramírez Pablo - Carlos Quintanilla Yerena

Drèze, Jean-Sen, Amartya-Hussein, Athar (1995). The Political Economy of Hunger. Great Britain: Clarendon Press.

Engels, Federico (1971). Prefacio a la situación de la clase obrera en Inglaterra. En: Marx, Carlos- Engels, Federico.Obras Escogidas, tomo II. Moscú: Editorial Progreso.

Friedman, Milton (1966). Capitalismo y libertad. Madrid: Ediciones RIALP.

Furniss, Edgar Stephenson (1965). The position of the laborer in a system of nationalism: a study in the labor theories of the later english mercantilists. New York: Augustus M. Kelley.

Hernández, Daniel-Orozco, Mónica-Vázquez, Sirenia (2005). La focalización como estrategia de politica pública. [Documentos de investigación, 25]. México: Secretaría de Desarrollo Social. México.

Himmelfarb, Gertrude (1998). La idea de la pobreza. Inglaterra a principios de la era industrial. México: Fondo de Cultura Económica.

La entrega de los apoyos monetarios de Progresa. Asequible en: http://cca.org.mx/ $\mathrm{cca} /$ web/ventana/ligas/monetarios_progresa.htm, fecha de consulta: 15-06-2019.

Nussbaum, Martha C.-Sen, Amartya (1998). La calidad de vida. México: Fondo de Cultura Económica.

Programa de Desarrollo Humano Oportunidades, montos mensuales correspondientes al segundo semestre de 2007. Asequible en: http://www.oportunidades.gob.mx/ informacion_general/Semestre_2_2007.pdf, fecha de consulta: 22-08-2018.

Salazar Silva, Fernando (2005). La configuración del Estado de Bienestar. Elementos constitutivos. Reflexión Politica, 14. 126-140.

Scott, John (1999). Análisis del Progresa: México. Experiencias exitosas de combate a la pobreza rural: Lecciones para una reorientación de las políticas [Informe final]. México: CIDE, Estudio RIMISP-FAO.

Sugden, Robert (1982). On the economics of philanthropy. The Economic Journal, 366. 341 350 .

Villarespe Reyes, Verónica (2002). Pobreza: teoría e historia. México: Juan Pablos-IIEcUNAM.

Villarespe Reyes, Verónica (2000). Los economistas políticos clásicos: pobreza y población. Algunos de sus teóricos relevantes. Problemas del Desarrollo, 123. 9-31.

Villatoro, Pablo (2004). Programas de reducción de la pobreza en América Latina. Un análisis de cinco experiencias. Santiago de Chile: CEPAL. Serie Políticas Sociales, 87. 